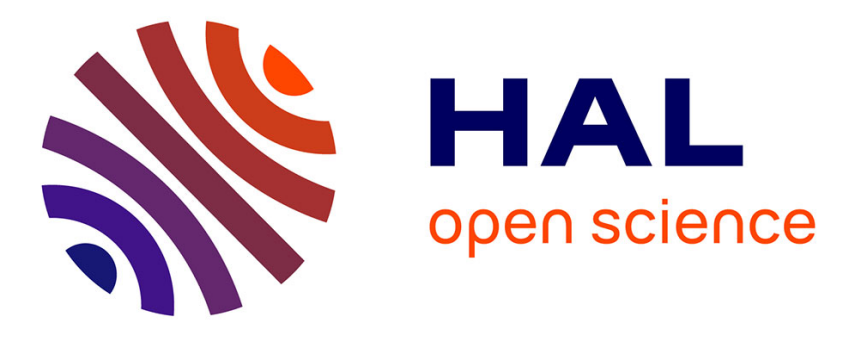

\title{
Design of Piezoelectric Actuators By Optimizing the Electrodes Topology
}

\author{
Thomas Schlinquer, Abbas Homayouni, Micky Rakotondrabe, Abdenbi \\ Mohand Ousaid
}

\section{- To cite this version:}

Thomas Schlinquer, Abbas Homayouni, Micky Rakotondrabe, Abdenbi Mohand Ousaid. Design of Piezoelectric Actuators By Optimizing the Electrodes Topology. IEEE Robotics and Automation Letters, 2020, 6 (1), pp.72-79. 10.1109/LRA.2020.3030561 . hal-02993825

\section{HAL Id: hal-02993825 \\ https://hal.science/hal-02993825}

Submitted on 10 Nov 2020

HAL is a multi-disciplinary open access archive for the deposit and dissemination of scientific research documents, whether they are published or not. The documents may come from teaching and research institutions in France or abroad, or from public or private research centers.
L'archive ouverte pluridisciplinaire HAL, est destinée au dépôt et à la diffusion de documents scientifiques de niveau recherche, publiés ou non, émanant des établissements d'enseignement et de recherche français ou étrangers, des laboratoires publics ou privés. 


\title{
Design of piezoelectric actuators by optimizing the electrodes topology
}

\author{
Thomas SCHLinguer $^{1}$, Abbas Homayouni-Amlashi ${ }^{1,2}$ \\ Micky RaKotondrabe ${ }^{2 *}$, member, IEEE and ,AbdenbiMohand-Ousaid ${ }^{1}$
}

\begin{abstract}
Piezoelectric materials based actuators are highly recognized for the development of microrobotic systems thanks to their high bandwidth, high resolution and high force density. However, one of their main drawback is the low relative stroke $(0.1 \%$ of actuator's size) that limits the actuator motion range. Overcoming this limitation is challenging but would increase the achievable working space of microrobots. In this paper, topology optimization method is used to maximize the actuator stroke. Instead of optimizing only the material density, we also consider the optimization of the electrodes polarity. This approach allows to combine both material expansion and compression in order to increase the actuator output displacement. To demonstrate this approach, two actuators were designed starting from a full domain considered as a basic reference piezoelectric actuator. The first design considered only the density optimization while the second one took into account the optimization of the topology of electrodes. Both simulation and experiment showed a good agreement between the obtained designs and the fabricated prototypes. The result revealed that the optimized design with polarization has an improved factors of 2.8 and 2.02 compared to full plate and actuator without electrodes optimization, respectively.
\end{abstract}

Index Terms-piezoelectric actuators, topology optimization, polarity optimization.

\section{INTRODUCTION}

Accurate and precise positioning is a key element to enhance the capability of microrobotic systems to manipulate and characterize objects in the microworld, i.e. the world of submillimetric objects [1]. The literature tells us how smart or active materials are widely recognized to the development of actuators for these microrobots and improve their accuracy [2]. These active materials are capable to change their physical inherent properties in response to an external stimuli like electrical field, magnetic field, thermal gradient or variation. Such feature allowed them to be largely employed as easily integrated and miniaturized actuators in the above microrobotic systems. Among these materials, piezoelectric materials are of particular interest since they can be used as an elementary actuator with high displacement resolution, large output force, high dynamics response and significant scaling-down possibilities [2] [3] [4]. However, due to their crystalline arrangement, they provide a low relative deformation ( $0.1 \%$ of actuator's size) that restricts

1 FEMTO-ST Institute, Université Bourgogne Franche-Comté / CNRS,Besançon - France.

${ }^{2}$ LGP laboratory, National School of Engineering in Tarbes (ENIT-INPT), University of Toulouse, Tarbes - France.

*: correponding author, abdenbi.mohandefemto-st. fr.

This work was supported by the national CODE-TRACK project (ANR17-CE05-0014-01, Control theory tools for optimal design of piezoelectric energy harvesters devoted to birds tracking devices) and was partially supported by the Bourgogne Franche-Comté region COMPACT project. their motion range [5]. Overcoming this limitation would increase the achievable working spcaes of the microrobotic systems in which they are integrated. As reviewed in [6], empirical and systematic approaches were investigated to the development of piezoelectric actuators. In particular several solutions have been proposed to increase their displacements. These solutions can be classified in four major categories: (i) staking piezoelectric layers, (ii) hybridization, (iii) amplification mechanisms, (iv) structural optimization.

The first class represents the trivial solution that operates on the shape, the polarity and the geometric arrangement of the actuator. It leads to piezostacks or multimorph configurations [7]-[9]. Piezostacks are the result of stacking several layers in serial in order to increase the output displacement. However to produce reasonable output displacement, this configuration leads to long and non-compact actuators. Multimorphs consist of several piezoelectric cantilevers layers. They provide larger displacement than piezostacks but at the expense of the generated force. In the hybridization class, the actuator is based on the combination of piezoelectric effect and an additional principle. Thermopiezoelectric actuators were mainly devoloped to this aim [10], [11]. The third class operates on external mechanism to amplify the actuator stroke. Known as amplifying mechanism, several structures have been investigated. The widespread ones are flexural lever structure [12] and flextensional mechanisms based on empirical or optimized structures such as Moonie [13] [14], Cymbal [15], nested rhombus structure [16] and optimal amplification mechanisms based on passive material [17], [18]. Despite their potential, these approaches only deal with geometrical or topological considerations of the amplification mechanism surrounding a predetermined actuator. In general, the amplifying mechanisms which are augmented to the actuators make the whole design bulky and heavy and inappropriate for small scale or micro dimension applications. Finally, the fourth class (structural optimization) is a systematic approach and includes parametric and topology optimization that consider piezoelectric materials within the optimization process [19]-[24]. The approach consists to find the actuator's optimal dimensions or shapes on the basis of intervals techniques [25] or with topology optimization methods [26] respectively. The advantage of the topology optimization is that there is no restriction on the shape of the actuator. Therefore, the whole structure of the actuator can be optimized. Although topology optimization provides a great degree of freedom in terms of structural optimization, the obtained results might bring complexity in terms of fabrication of the designed structures. However this challenge 
can be tackled by defining proper optimization parameters as it is explained in [26]. One of the used topology optimization methods to integrate piezoelectric materials is the SIMP (Solid Isotropic Material with Penalization) method [27]. For instance it was successfully applied to design piezoelectric structures such as gripper [28]. Designed as a monolithic structure, the achievement has shown a quite good performance by maximizing the in-plane displacement at the output of the gripper, whilst the out-of-plane bending of the gripper's jaw was suppressed. In spite of these interesting and promising achievements, this approach considers only the piezoelectric material distribution while the polarity of electrodes is kept fix.

In this paper, we propose to design piezoelectric actuators with the SIMP method. In addition, not only the density is optimized but we propose a modification of the algorithm such that the structure polarity is also considered. Such consideration is useful for two reasons: (i) it provides several degrees of freedom in terms of optimization where two variables (density and polarity) can be considered, and (ii) it allows to combine material expansion and compression in order to increase the output displacement. Therefore, the proposed approach will optimize the structure of the actuator without augmenting any additional structure. In this case, the optimized designs can be miniaturized for microrobotic applications. To validate the proposed approach, two actuators will be designed where their output displacements are maximized. The design of each actuator starts from a full domain plate considered as a basic reference actuator. Within this domain, the first design is based on density optimization with a fix polarization of the electrodes, whilst the second design is based on both density and polarity optimization. The result will reveal that when polarity is considered, the outcome actuator provides 2.8 and 2.02 as amplification factors in term of displacement compared to full plate and actuator without electrode optimization, respectively. Simulations and experiments are performed to validate the obtained designs and the fabricated prototypes.

\section{TOPOLOGY OPTIMIZATION OF PIEZOELECTRIC STRUCTURES}

This section briefly reminds (i) the principle of topology optimization (ii) the main major steps allowing to derive finite element model of a thin piezoelectric plate and (iii) the integration of piezoeletric model within the topology optimization method.

\section{A. Topology optimization}

Topology optimization especially SIMP method is a design methodology aiming to find an optimal structure within a limited design domain [26]. Based on material distribution, it consists to minimize systematically an objective function while subjected to one or several constraints. To this end, the method combines finite element formulation and penalization power law to make material intermediate density unattractive and therefore avoid the $0-1$ problem of classical topology optimization processes. Presented in [26], SIMP method is

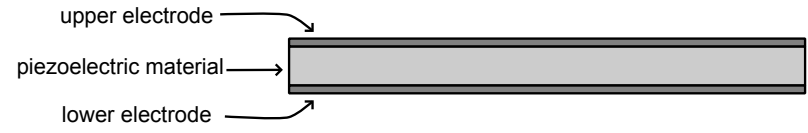

Fig. 1. Piezoelectric material sandwiched between electrodes.

easy to implement and suitable for the design of passive structures. Since becoming a conceptual tool for structural design, it has been successfully applied for topological design of active structures in particular piezoelectric structures [23], [24]. This methodology made possible the consideration of the physics of the material within the optimization problem. To do so, the finite element model of the piezoelectric structure is required which we provide in the next section.

\section{B. Piezoelectric modeling of $2 D$ thin plate}

As we target the design of $2 \mathrm{D}$ actuators, a plate model is considered in this paper. It consists of a thin piezoelectric layer sandwiched between two electrodes as illustrated in Fig. 1. This configuration is widely used as a framework for the design of planar active mechanism. To derive its model, we first assumed $\vec{z}$ the normal axis of the thin plate which is considered to be the axis of polarization as well. Then, by applying the plane-stress theory [29], the nominal stresses perpendicular to the xy plane are zero. We chose a $4 \mathrm{~mm}$ class tetragonal crystal of piezoelectric materials [30] to derive the corresponding model. This class includes most of the piezoelectric material in particular the well-known PZT material [31]. According to these hypothesis, the "e" form constitutive equations from [32] are simplified to obtain the following new set of relations:

$$
\left\{\begin{array}{l}
T_{1} \\
T_{2} \\
T_{6} \\
D_{3}
\end{array}\right\}=\left[\begin{array}{cccc}
c_{1} & c_{2} & 0 & -e \\
c_{2} & c_{1} & 0 & -e \\
0 & 0 & c_{6} & 0 \\
e & e & 0 & \varepsilon
\end{array}\right]\left\{\begin{array}{l}
S_{1} \\
S_{2} \\
S_{6} \\
E_{3}
\end{array}\right\}
$$

where:

$$
\begin{gathered}
c_{1}=c_{11}^{E}-\frac{\left(c_{13}^{E}\right)^{2}}{c_{33}^{E}}, c_{2}=c_{12}^{E}-\frac{\left(c_{13}^{E}\right)^{2}}{c_{33}^{E}}, c_{6}=c_{66}^{E} \\
e=e_{31}-e_{33} \frac{c_{13}^{E}}{c_{33}^{E}}, \varepsilon=\varepsilon_{33}^{S}+\frac{\left(e_{33}\right)^{2}}{c_{33}^{E}}
\end{gathered}
$$

The mechanical coefficients of the tensor given in equation (1) can be rewritten by identifying them with the plane stress isotropic tensor [29]:

$$
[C]=\frac{E}{1-v^{2}}\left[\begin{array}{ccc}
1 & v & 0 \\
v & 1 & 0 \\
0 & 0 & \frac{1-v}{2}
\end{array}\right]
$$

Therefore, we express the mechanical stiffness tensor of the plan-stress form of constitutive equation in (1) by Young's modulus and Poisson's ratio. Considering the $4 \mathrm{~mm}$ piezoelectric class property where $c_{6}=\frac{1}{2}\left(c_{1}-c_{2}\right)$ [32], the Young's modulus $E$ and Poisson's ratio $v$ according to $c_{1}$ and $c_{2}$ can be expressed as follows: 


$$
E=\frac{c_{1}^{2}-c_{2}^{2}}{c_{1}}, v=\frac{c_{2}}{c_{1}}
$$

Taking advantage of planar isotropy of $4 \mathrm{~mm}$ crystal, the planar Young's modulus and Poisson's ratio of this class of piezoelectric material can be calculated. This is an essential step for the normalization process which will be discussed in the next sections. In the rest of the paper, we consider the commercial piezoelectric material PSI-5H4E from Piezo Systems Inc. By substituting its numerical values, we obtain $E=66.2 \mathrm{GPa}$ and $v=0.298$. For the piezoelectric and dielectric coefficients, we obtain $e=-28.30 \mathrm{C} . \mathrm{m}^{-1}$ and $\varepsilon / \varepsilon_{0}=1932$ with $\varepsilon_{0}$ the vacuum's permittivity.

\section{Finite element model of thin piezo plate}

The finite element model of the piezoelectric plate can be derived by following the general approach given in [33]. It consists to compute the stiffness matrices using the thin plate model described previously and results in the equilibrium equation of the piezoelectric material. For this purpose, we extend the bilinear 4 nodes element to take into account the piezoelectric behavior. In this case, four classical nodes model the mechanical behavior while two additional nodes model the electrical degree of freedom along the $\vec{z}$ that correspond to the electrodes. Each element is considered as a flat cuboid with side length $l$ and thickness $h$. Based on this configuration, interpolation functions for both displacement and electrical potential are defined as follows:

$$
N_{u}^{i}=\frac{(l \pm 2 x)(l \pm 2 y)}{4 l^{2}}, N_{\phi}^{j}=\frac{h \pm 2 z}{2 h}
$$

where $i$ and $j$ index each node, either mechanical or electrical. Based on these functions, we compute the mechanical and piezoelectric stiffness matrices following the procedure described in [33]:

$$
\begin{gathered}
{\left[K_{u u}\right]=k_{0}\left[\widetilde{K}_{u u}\right], k_{0}=\frac{E h}{1-v^{2}}} \\
{\left[K_{u \phi}\right]^{T}=\frac{l e}{2}\left[\begin{array}{cccccccc}
-1 & -1 & 1 & -1 & 1 & 1 & -1 & 1 \\
1 & 1 & -1 & 1 & -1 & -1 & 1 & -1
\end{array}\right]}
\end{gathered}
$$

with $\left[\widetilde{K}_{u u}\right]$ is well-known dimensionless eight by eight matrix (see [34] for more details) and $k_{0}$ is the normalization factor which was calculated with the help of the derived Young modules and Poisson's ratio. Using these two matrices, the global finite element system for actuation is expressed as:

$$
\{F\}=\left[K_{u u}\right]\{U\}+\left[K_{u \phi}\right]\{\phi\}
$$

where $F$ and $U$ are respectively the nodal force and displacement vectors. Unlike passive material, $F$ here is null since the force is generated by the material itself, i.e no external load force. The above equation can be simplified knowing that $\{\phi\}^{T}=\left\{\begin{array}{ll}\phi_{1} & \phi_{2}\end{array}\right\}$ and the potential $V=\phi_{1}-\phi_{2}$ :

$$
\begin{aligned}
\left(\left[K_{u \phi}\right]\{\phi\}\right)^{T} & =\frac{V l e}{2}\left\{\begin{array}{llllllll}
-1 & -1 & 1 & -1 & 1 & 1 & -1 & 1
\end{array}\right\} \\
& =f_{0}\left\{\widetilde{F}_{u \phi}\right\}^{T} \text { where } f_{0}=\frac{\text { Vle }}{2}
\end{aligned}
$$

where $f_{0}$ is the normalization factor of the piezoelectric matrix. With this simplification, equation (9) can be written as:

$$
\{0\}=k_{0}\left[\widetilde{K}_{u u}\right]\{U\}+f_{0}\left\{\widetilde{F}_{u \phi}\right\}
$$

Here $k_{0}$ stands for a mechanical stiffness while $f_{0}$ represents the internal force generated by the material when subjected to voltage. For simplification, we denote in the rest of the paper $\left[\widetilde{K_{u u}}\right]$ as $\left[K_{n}\right]$ and $\left\{\widetilde{F_{u \phi}}\right\}$ as $\left\{F_{n}\right\}$, where subscript $n$ stands for normalization. This last term can be interpreted as the acting of piezoelectric forces on the element. This leads to a simple and compact finite element equation:

$$
k_{0}\left[K_{n}\right]\{U\}=-f_{0}\left\{F_{n}\right\}
$$

By analogy to pure mechanical behavior, this finite element model defines the equilibrium equation of a piezoelectric material which is mandatory to solve the topology optimization problem. Finally, the displacement vector is normalized for numerical considerations:

$$
\begin{aligned}
k_{0}\left[K_{n}\right]\{U\}=-f_{0}\left\{F_{n}\right\} & \Leftrightarrow\left[K_{n}\right]\left(-\frac{k_{0}}{f_{0}}\right)\{U\}=\left\{F_{n}\right\} \\
& \Leftrightarrow\left[K_{n}\right]\left(-\frac{1}{u_{0}}\right)\{U\}=\left\{F_{n}\right\} \\
& \Leftrightarrow\left[K_{n}\right]\left\{U_{n}\right\}=\left\{F_{n}\right\}
\end{aligned}
$$

Such normalization has two important consequences: (i) the equation is independent from the material coefficients, domain dimensions and input voltage, which greatly improves the element versatility. The physical displacement can still be obtained using the elemental displacement $u_{0}$. It is worth to notice that we are still dependent on the Poisson's ratio through the stiffness matrix, (ii) each member of equation (15) is of an order of magnitude between -2 and 0 that helps to reduce rounding error when resolving the linear system described by this equilibrium equation.

\section{Integration of piezoelectric material within topology op- timization method}

Based on the above-derived finite element model, two modifications are brought in order to implement this model within SIMP method. The first modification concerns the extension of the classical penalization scheme that considers both density and polarity variables, whilst the second relies on the reformulation of the objective function and its derivation in respect to these two variables.

1) SIMP penalization scheme extension: Following the original penalization scheme presented in [26], several extensions have been proposed to include piezoelectric material [27], [35]-[39]. Most of them were applied on the material properties tensor $\left[c^{E}\right],[e]$ and $\left[\varepsilon^{S}\right]$. However, these schemes penalize each coefficient of the tensors at the expense of computational time. To tackle this limitation, we propose to apply the penalization directly as a coefficient in front of the normalized stiffness matrix and force vector. This leads to a simple penalization scheme which can be written 
according to the material density $\rho$ and polarization direction value $p_{e}$ as follows:

$$
\begin{aligned}
& {\left[K_{n}\right](\rho)=k_{p}(\rho)\left[K_{n}\right]} \\
& \left\{F_{n}\right\}(\rho)=k_{f}\left(\rho, p_{e}\right)\left\{F_{n}\right\}
\end{aligned}
$$

with

$$
\begin{aligned}
k_{p}(\rho) & =\left(k_{p}-k_{\text {min }}\right) \rho^{p_{k}}+k_{\text {min }} \\
k_{f}\left(\rho, p_{e}\right) & =\left(f_{p}-f_{\text {min }}\right)\left(2 p_{e}-1\right) \rho^{p_{f}}+f_{\text {min }}
\end{aligned}
$$

where $p_{k}$ and $p_{f}$ are the stiffness and force penalization coefficients and $k_{\text {min }}$ and $f_{\text {min }}$ are the minimum stiffness and force factors. These minimum values are defined to avoid the singularity problems during the optimization [26] [40] [41]. The consideration of polarization direction as a variable is introduced in a methodology known as "piezoelectric material with penalization and polarization" (PEMAP-P) [27].

To apply this new penalization scheme, all parameters must be known. For $k_{p}, f_{p}, k_{\min }$ and $f_{\min }$, we use the recommended numerical values $\left(k_{p}=f_{p}=1\right.$ and $k_{\text {min }}=$ $\left.f_{\min }=10^{-9}\right)$ given in [26]. Material Density $(\rho)$ and polarization direction $\left(p_{e}\right)$ can have values between zero and one. By introduction of penalization coefficients $\left(p_{k}, p_{f}\right)$, the material density $(\rho)$ will steer to zero (void) or one (material) during the optimization iterations. However, the penalization coefficients are difficult to estimate since there is no analogy with pure mechanical behavior. Beside, the fact that the penalization coefficients need to be higher than one [26], there is no computation of those coefficients for plane stress heterogeneous piezoelectric material to the knowledge of the authors. This challenging issue remains an active research domain as shown in [42]-[44]. In this work we set both penalization factors constant to a value of three and no penalization coefficient is defined for the polarization direction since the best value for it is one [27]. Giving rise to consistent results, we did not investigate further this issue.

2) Objective function reformulation: Optimizing piezoelectric actuators can be done following several objectives like electric-mechanical conversion [37] or optimal displacement [27]. For simplicity, only the latter is considered in this paper. Following the classical mechanical approach reported in [26], we rewrote the objective as a minimization of an objective function $J_{\text {out }}=-u_{\text {out }}=-L^{T} U_{n}$ where $L$ is a vector with a value of one that corresponds to the output displacement node and zero otherwise. However, while the variation of this objective function reflects the evolution of the optimization process, its value holds no meaning due to the normalization of the finite element system. The elementary displacement $u_{0}$ could be used, but this would nullify the versatility of the approach. Instead, we propose to normalize the objective function using its value when the domain is not yet optimized. This initial objective function value is now named $J_{0}$. This has an immediate consequence that allows to understand the objective as how much more displacement can be obtained by the optimized design comparing to full domain. This new objective function can be written as $J=\frac{J_{\text {out }}}{J_{0}}=-L^{T} U_{n}$ where the vector $L$ includes the normalization factor $J_{0}$.
To perform a gradient based optimization, the sensitivities of this objective function with respect to material density $(\rho)$ and polarization $\left(p_{e}\right)$ are calculated using the well-known adjoint method. In addition, we wrote the system of equation (15) as $K U=-F$ and the objective function as $J=-L^{T} U$ in order to simplify the notations.

$$
\begin{aligned}
\frac{\partial J}{\partial \rho} & =\frac{\partial}{\partial \rho}\left(-L^{T} U+\Lambda^{T}(K U+F)\right) \\
& =\frac{\partial}{\partial \rho}\left(\left(-L^{T}+\Lambda^{T} K\right) U+\Lambda^{T} F\right) \\
& =\Lambda^{T} \frac{\partial K}{\partial \rho} U+\left(-L^{T}+\Lambda^{T} K\right) \frac{\partial U}{\partial \rho}+\Lambda^{T} \frac{\partial F}{\partial \rho} \\
& =\Lambda^{T}\left(\frac{\partial K}{\partial \rho} U+\frac{\partial F}{\partial \rho}\right)
\end{aligned}
$$

We obtain the last simplification by specifying $-L^{T}+$ $\Lambda^{T} K=0$. This system can be rewritten as $K \Lambda=L$ using the property $K=K^{T}$, hence $\Lambda$ can be seen as a displacement field resulting from a dummy force $L$ which is applied at the output displacement node [26]. Following the same procedure, the sensitivity of objective function with respect to polarization can be obtained:

$$
\frac{\partial J}{\partial p_{e}}=\Lambda^{T}\left(\frac{\partial F}{\partial p_{e}}\right)
$$

The main difference with pure mechanics is the dependency on the sensitivities of the piezoelectric force $\frac{\partial F}{\partial \rho}$ and $\frac{\partial J}{\partial p_{e}}$. The stiffness matrix sensibility can be directly computed as follows:

$\frac{\partial K}{\partial \rho}=\frac{\partial}{\partial \rho}\left(k_{p}\left[K_{n}\right]\right)=\frac{\partial k_{p}}{\partial \rho}\left[K_{n}\right]=p_{k}\left(k_{p}-k_{\text {min }}\right) \rho^{p_{k}-1}\left[K_{n}\right]$

The same procedure is applied for the piezoelectric force sensibility:

$$
\begin{aligned}
\frac{\partial F}{\partial \rho} & =p_{f}\left(f_{p}-f_{\text {min }}\right)\left(2 p_{e}-1\right) \rho^{p_{f}-1}\left\{F_{n}\right\} \\
\frac{\partial F}{\partial p_{e}} & =2\left(f_{p}-f_{\text {min }}\right) \rho^{p_{f}}\left\{F_{n}\right\}
\end{aligned}
$$

This last step complete the integration process of the piezoelectric material within the SIMP method. In terms of programming, we re-use and fit the 88 lines code [34] in order to include the 2D finite element presented above. Then, the OC (Optimal Criteria) is substituted with the MMA (Method of Moving Asymptotes) in order to improve the stability and performances of the optimization algorithm.

\section{Optimal DESIGN OF PIEZOELECTRIC ACTUATORS}

This section focuses on the design of one degree of freedom piezoelectric actuators using the approach described previously. Two actuators are designed in order to highlight the importance of optimizing the electrodes topology. The first actuator was based on density optimization with uniform electrodes while the second is based on the combination of density and polarity optimization. 


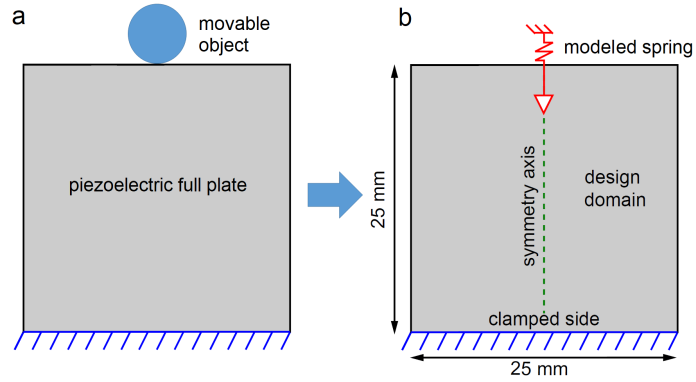

Fig. 2. Pusher geometric and mechanical specification. Gray area represents the design domain

\section{A. Designs specification}

Figure 2.a illustrates the initial optimization domain set to design both actuators. It consists on a square plate of dimensions $25 \mathrm{~mm} \times 25 \mathrm{~mm} \times 127 \mu \mathrm{m}$. Within this domain, the middle point of the top edge is chosen as the actuator tip. Thus the objective is to maximize the displacement of this point when optimizing the design. According to section IID.2, we proceed by modeling this output as a force in the opposite direction and an artificial spring with low stiffness in comparison to piezoelectric material itself, as shown in red in Fig. 2.b. This is a well known procedure in designing the compliant mechanisms and further details can be found in [26]. We consider the stiffness of the spring to be 0.01 of the derived young modulus of the piezoelectric material in section II-B. Furthermore, we model the clamping of the plate by adding supports at the bottom edge of the domain (blue boundary). At this stage, these specification are the same for both designs. For the first design a fixed uniform voltage over the upper electrode is considered and the lower electrode is grounded. In contrast, the second design considers electrodes polarity as an optimization variable. In this case, the electrodes can be set to ground, positive or negative potential depending on the optimization process. Finally, we take advantage of the symmetry to have the entire working domain in order to reduce the computation time and memory usage.

To solve this problem, we start by discretizing the design domain using the finite element approach presented in Sec. II. The size of each element is set to $200 \mu \mathrm{m}$, resulting in a mesh of $125 \times 250$ elements. Finally, the volume fraction is set to 0.3 , meaning that only $30 \%$ of the full domain will be retained for the optimized design.

\section{B. Post-processing}

The obtained designs after optimization can be seen in Fig. 3.a and Fig. 4.a. The efficiencies (output mechanical energy/input electrical energy [45]) of the full piezoelectric plate, optimized design without polarity optimization and with polarity optimization are $0.482,0.474$ and 0.445 respectively. A negligible decrease in the efficiency of the optimized design can be seen. However, the details of force and displacement should be investigated after post processing. Although the raw designs are already well defined, we apply a classic post-processing approach to ease the exportation of the design as CAD model. First, the resolution is increased by a factor of three using bilinear interpolation. This operation allows to perform an anti-aliasing effect by smoothing the boundaries for cleaner exportation results. Next, a volume preserving threshold is performed to convert the intermediate binary densities, while maintaining the global volume of the domain. This is done using a simple dichotomy approach to find the optimal threshold. Doing so, it is easy to export the design to CAD software following the afterwards steps: (i) perform a contour detection of the post processed design under Matlab, (ii) export the resulted contour under Space Claim software from ANSYS (ANSYS, Inc, Canonsburg, USA) as a cloud of point, (iii) interpolate the sketch using splines and extrude the thickness of the piezoelectric plate. Then, with the help of the obtained CAD files, the performances of the optimized designs are analyzed with the commercial finite element software COMSOL Multiphysics (COMSOL, Inc.,Burlington, USA)

\section{Simulation}

Figure 3.a presents the result of the topology optimization algorithm for the case of no polarization optimization. Different interesting features can be observed from the result. The overall structure can be described as a three members lattice beams. When powered, the beams straighten, increasing their length. As a result, two amplification effects are created. First, the beams on the left side play a role of mechanical lever that converts the symmetrical extension to horizontal movement by rotation. Second, the right beam acts as an extension rod, further pushing forward. This behavior is well illustrated in Fig. 3.b presenting the displacement field norm superposed with the resulting deformed domain. We conclude on the raw design by evaluating its performance as presented in section II-D. 2 resulting in an amplification ratio of 1.38 , i.e. we have obtained $+38 \%$ displacement than a full plate of the same dimensions.

The result of the topology optimization with polarization optimization is shown in Fig 4.a. In this figure, the working principle of the grey areas which have the same polarization direction is similar to the one of previous result without polarization optimization. However, the major difference is the part with inverse polarization direction. With the same applied voltage, this part will be retracted and the combination of retraction and extension in the design will result to considerable improvement of displacement amplification. For example, the simulation in Fig 4.b shows that displacement of the optimized design with optimization of the polarity is almost twice the displacement of the one without the optimization of the polarity in Fig. 3 .

The numerical data of simulation is reported in Table I. In this table the displacement per volt, the blocking force, energy density and the related gains for an applied voltage of 5 volt are reported. Energy density is the mechanical energy divided by piezoelectric mass. The mechanical energy is calculated as presented in [22]. As can be seen in Table I, the displacement gains with respect to full plate for 


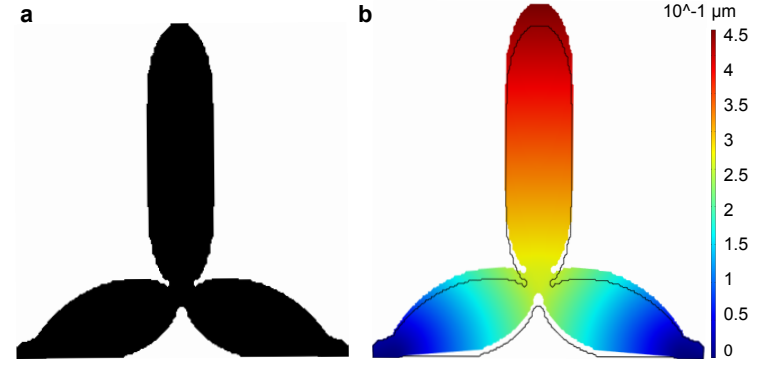

Fig. 3. Optimized design without polarization optimization. a) The obtained layout by optimization b) COMSOL simulated deformation after applying $5(\mathrm{~V})$ actuation voltage.

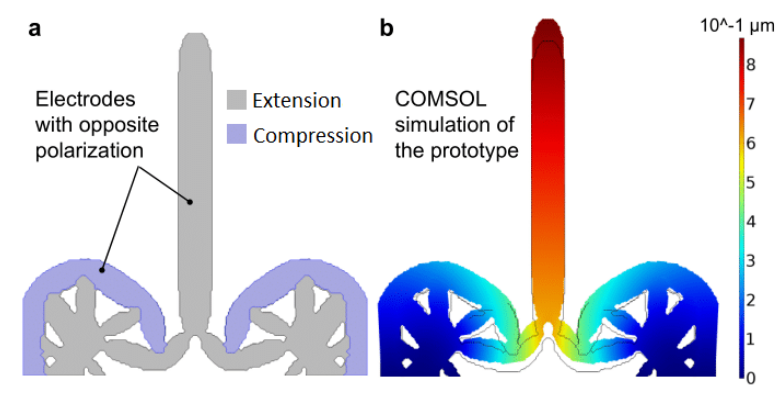

Fig. 4. Optimized design with polarization optimization. a) The obtained layout by optimization b) COMSOL simulated deformation after applying $5(\mathrm{~V})$ actuation voltage.

optimized design without polarization and with polarization optimization are 1.42 and 2.82 respectively. On the other hand, the gains of blocking force are decreasing by factors of 0.66 and 0.33 respectively. This is expected since increasing the displacement is always accompanied by reduction of output force. However, it should be noted here that optimized designs have only 30 percent of the volume of the full plate. Therefore, a great improvement in energy density can be seen for optimized designs as it is reported in Table I.

\section{EXPERIMENTAL VALIDATION}

\section{A. Prototype fabrication}

The fabrication process of the prototypes start by converting the optimzed designs to an exploitable CAD model. Based on the CAD model, a Starlight laser engraving machine from SiroLasertec (Siro Lasertec GmbH, Pforzheim, Germany) is used to achieve cutting operations. Starting from a piezoelectric full plate with a dimensions of 70x70x0.127 mm (from Piezo Systems), the three prototypes shown in Fig. 5(a,b,c) were fabricated: (a) full plate, (b) prototype without optimized polarity and (c) prototype with optimized polarity. For the last one, two cutting operations were necessary. The first one consisted to cut the main structure of the actuator while the second consisted to separate the electrodes following the optimized electrode profile. It is worth to note that a rectangle domain of $5 \mathrm{~mm}$ was added on the resulting design. This extension allows to fix the design on a custom made PCB to ensure mechanical clamping. Furthermore, silver-based conductive glue was used to ease the electrical connection with the lower and upper electrodes.
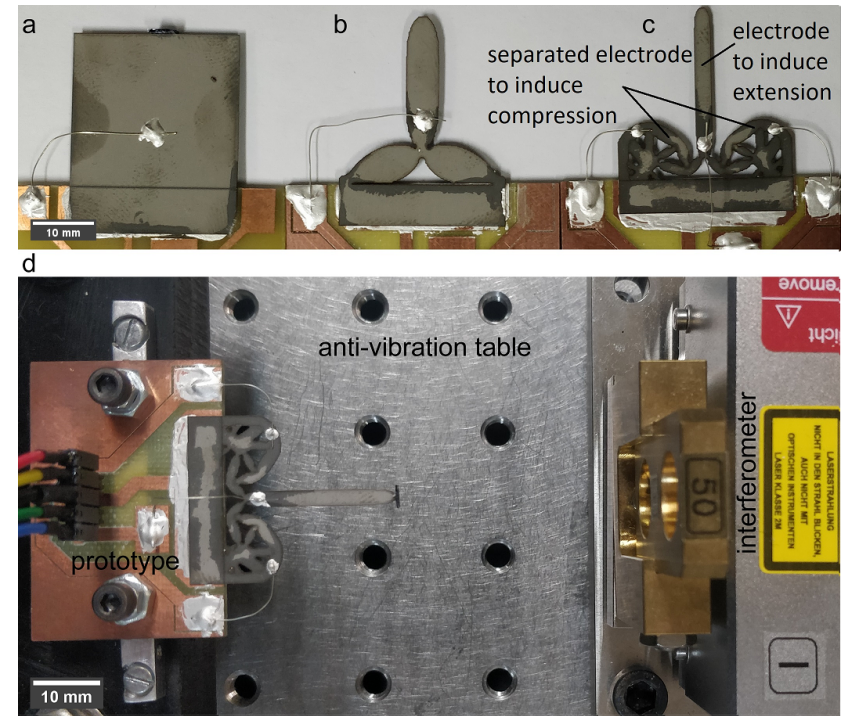

Fig. 5. Fabricated prototypes and experimental bench. a) full plate actuator, b) actuator prototype without optimized polarity, c) actuator prototype with optimized polarity following the profile of Fig. 4 (a), d) general view of the experimental set-up.

\section{B. Experimental Bench}

Figure 5.d shows the experimental set-up used to characterize the prototype. It is composed of:

- an interferometer (SIOS, GmbH company) able to measure displacements (deformations) with nanometric resolution,

- the prototypes to be characterized and fixed on PCB supports,

- an acquisition system (computer with Matlab + dSPACE board) to generate excitation signals, and to record, process and analyze measured experimental data.

The prototypes and the interferometer probe were placed on an anti-vibration table to avoid vibrations that may come from the ground.

\section{Characterization of Results and Discussion}

The objective of experimental investigation is to measure the displacements in order to demonstrate the efficiency of optimized designs in comparison to full plate and to validate the results obtained in the simulation part. We use sine voltage signals with low amplitude in order to respect linearity assumption of the modelling. A series of measurements is performed under an excitation that goes from $0 \mathrm{~V}$ to $5 \mathrm{~V}$ with a step of $0.5 \mathrm{~V}$ and a frequency of $2 \mathrm{~Hz}$. This frequency is low enough in comparison with the plate and prototypes natural frequency such that the static model of the system which was presented in section II-C is respected. The domain of applied voltage is chosen to keep the material behavior only in its linear domain, as the model used for the optimization is based on linear assumptions. Higher voltage and higher frequencies can result in nonlinear behavior as hysteresis [46]. Each series is applied alternatively on the full plate, prototype without polarization and prototype with polarization. The resulting average displacements are reported in Fig. 6 and 
TABLE I

SUMMARY OF SIMULATION AND EXPERIMENTAL DATA

\begin{tabular}{cccc} 
& \multicolumn{3}{c}{ Simulation ( Input voltage = 5V ) } \\
\cline { 2 - 4 } & Full plate & Opt without pol & Opt with pol \\
\hline Displacement $(\mathrm{nm} / \mathrm{V})$ & 57 & 81 & 161 \\
Displacement gain w.r.t.f.p & - & 1.42 & 2.82 \\
Blocking force $(\mathrm{N})$ & 2.56 & 0.21 & 0.18 \\
Blocking force gain w.r.t.f.p & - & 0.08 & 0.07 \\
Energy density $\left(\mathrm{J} / \mathrm{m}^{3}\right)$ & 4.55 & 1.81 & 3.10 \\
Energy density gain w.r.t.f.p & - & 0.39 & 0.68
\end{tabular}

Experiment ( Input voltage $=5 \mathrm{~V}$ )

\begin{tabular}{cccc} 
& \multicolumn{3}{c}{ Experiment ( Input voltage = 5V ) } \\
\cline { 2 - 4 } & Full plate & Opt without pol & Opt with pol \\
\hline Displacement $(\mathrm{nm} / \mathrm{V})$ & 62 & 86 & 174
\end{tabular}

Displacement gain w.r.t.p

${ }^{\star}$ w.r.t.f.p : with respect to full plate

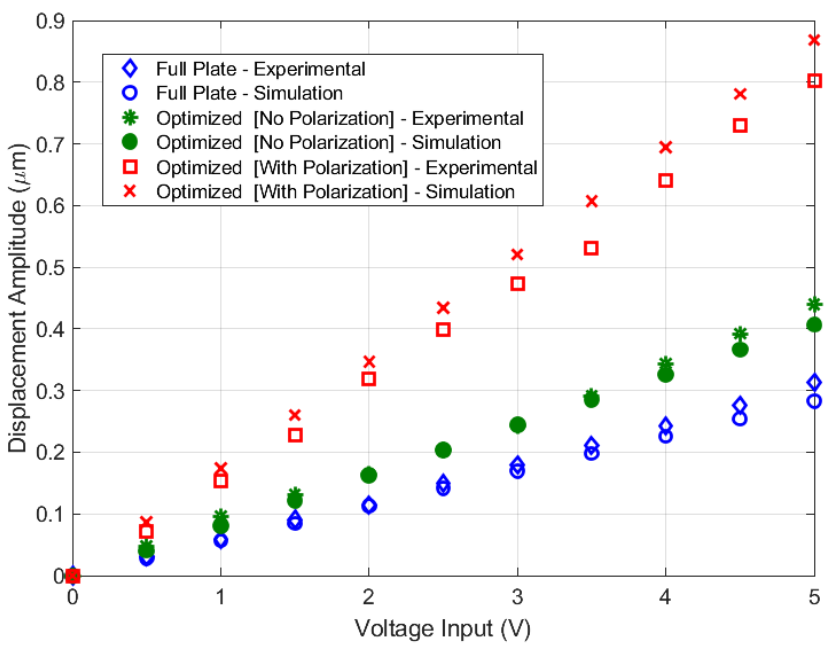

Fig. 6. Experimental characterization of prototypes.

Table I summarizes the obtained performance gains for both simulations and experimental validation.

First, based on Fig. 6, it is obvious that there is a satisfying agreement between the experimental and the simulation data. However by increasing the voltage, the difference between the experimental and the simulation data increases. This is due to the inherit nonlinear behavior of piezoelectric materials. Though, within the domain of the applied voltage, linear assumption in the modelling produces negligible error.

Then, Fig. 6 demonstrates better performances of the optimized designs with respect to full plate design. The improvement factor from the optimized design without polarization optimization is 1.38. On the other hand, the improvement factor from the optimized design with polarization optimization is close to 2.8. Hence, the optimized polarization design proposes 2 times more displacement than the nonoptimized polarization design. This improvement primarily proves that reducing the material volume and optimizing the topology of the structures within the structures domain can provide more flexible design in terms of producing the desired displacement. Furthermore, it is illustrated that polarization optimization allows both expansion and contraction within the design domain and thus amplifies the displacement amplitude significantly.

The results in Fig. 6 and Table I demonstrate two accom- plishments in term of precision and displacement. However, it is true that the displacement amplification is acquired by decreasing the output force of the actuator. Although the experimental measurements of the piezoelectric actuator's output force is highly challenging due to the low thickness to length ratio of the piezoelectric plate, the decrease in the output force is calculated by FEM simulation and it is reported in Table I. In fact, by increasing the stiffness of the modeled spring at the tip of the actuator, the optimization will produce a simple bar beam to maximize the force. On the other hand, the in-plane piezoelectric force is much higher than the out of plane force. Therefore, the output force of the optimized designs are still enough for the applications like atomic microscopy or in applications where the actuator stroke is essential.

In summary, the method allows to reduce drastically the material amount. Indeed, only $30 \%$ of the material was optimally distributed in order to provide a displacement greater than the displacement of an actuator with a uniform polarization. This leads to a compact and economical design. This is particularly interesting in the context of miniaturization, since the non-occupied space can be utilized to implement additional functionalities like sensors or electronic circuits.

\section{Conclusions And Perspectives}

In this paper, the optimal design of a piezoelectric actuators was presented. Based on topology optimization approach, the design improved the actuator maximum stroke comparing to conventional actuators, owing to the combination of material density and electrodes polarity optimization. To do so, first a finite element model of piezoelectric plate with the help of plane-stress assumption was established and a normalization was introduced to increase the generality of the proposed method. In addition, the polarization was considered as a variable of optimization to give further degree of freedom to the topology optimization algorithm for finding the optimal layout of the piezoelectric actuator. The objective function was defined to maximize the stroke of the actuator based on the defined stiffness of the movable object. To better investigate the effect of polarization, two optimized design with and without polarization optimization were obtained. The performance of these two optimized designs were investigated by numerical simulation and experimental validation. Satisfying agreement between the numerical and experimental results were observed. It was proved that optimization of polarization brings both expansion and contraction within the design domain which will magnify the amplitude of the displacement significantly. The stroke obtained from the optimized design with the polarization optimization is twice larger than that from the design without polarization optimization.

Future work extend the approach to take into account the out-of-plane displacement. Indeed, a large class of piezoelectric actuators use multilayered structure which benefit from large bending displacement in [47]. This extend will pave the way for the design of multi-degrees of freedom actuators. 


\section{REFERENCES}

[1] S. Régnier and N. Chaillet, Microrobotics for Micromanipulation. Wiley-ISTE, 2010.

[2] A. Kortschack and S. Fatikow, "Smart materials for actuation in microrobots," in Smart Structures, Devices, and Systems, E. C. Harvey, D. Abbott, and V. K. Varadan, Eds., vol. 4935, International Society for Optics and Photonics. SPIE, 2002, pp. $1-14$.

[3] S. Dong, "Review on piezoelectric, ultrasonic, and magnetoelectric actuators," Journal of Advanced Dielectrics, vol. 2, p. 1230001, 2012.

[4] H. McClintock, F. Z. Temel, N. Doshi, J.-s. Koh, and R. J. Wood, "The millidelta: A high-bandwidth, high-precision, millimeter-scale delta robot," Science Robotics, vol. 3, no. 14, 2018.

[5] M. Grossard, C. Rotinat-Libersa, N. Chaillet, and M. Boukallel, "Mechanical and control-oriented design of a monolithic piezoelectric microgripper using a new topological optimization method," IEEE/ASME Transactions on Mechatronics, vol. 14, no. 1, pp. 32-45, 2009.

[6] S. Wang et al., "A survey of piezoelectric actuators with long working stroke in recent years: Classifications, principles, connections and distinctions," Mechanical Systems and Signal Processing, vol. 123, pp. 591-605, 2019.

[7] D. L. DeVoe and A. P. Pisano, "Modeling and optimal design of piezoelectric cantilever microactuators," Journal of microelectromechanical Systems, vol. 6, no. 3, pp. 32-45, 1997.

[8] B. K. Seung-Yop Lee and W. Yang, "Theoretical modeling, experiments and optimization of piezoelectric multimorph," Smart Materials and Structures, vol. 14, pp. 1343-1352, 2005.

[9] L. L. Huajun Fang and T. Ren, "Modeling and design optimization of large-deflection piezoelectric folded cantilever microactuators," Transactions on Ultrasonics, Ferroelectrics, and Frequency Control, vol. 53, no. 1, pp. 1343-1352, 2006.

[10] M. Rakotondrabe and A. Ivan, "Development and dynamic modeling of a new hybrid thermo-piezoelectric micro-actuator," IEEE Transactions on Robotics, vol. 26, no. 6, pp. 1077-1085, 2010.

[11] M. Rakotondrabe and I. A. Ivan, "Development and force/position control of a new hybrid thermo-piezoelectric microgripper dedicated to micromanipulation tasks," IEEE Transactions on Automation Science and Engineering, vol. 8, no. 4, pp. 824-834, 2011.

[12] K. Uchino, "Electrostrictive actuators: Materials and applications," vol. 65, pp. 647-652, 041986.

[13] Y. Sugawara, K. Onitsuka, S. Yoshikawa, Q. Xu, R. E. Newnham, and K. Uchino, "Metal-ceramic composite actuators," Journal of the American Ceramic Society, vol. 75, no. 4, pp. 996-998, 1992.

[14] A. Dogan, Q. Xu, K. Onitsuka, S. Yoshikawa, K. Uchino, and R. E. Newnham, "High displacement ceramic metal composite actuators (moonies)," Ferroelectrics, vol. 156, no. 1, pp. 1-6, 1994.

[15] A. Dogan, K. Uchino, and R. E. Newnham, "Composite piezoelectric transducer with truncated conical endcaps "cymbal"," IEEE Transactions on Ultrasonics, Ferroelectrics, and Frequency Control, vol. 44, no. 3, pp. 597-605, 1997.

[16] J. Ueda, T. W. Secord, and H. H. Asada, "Large effective-strain piezoelectric actuators using nested cellular architecture with exponential strain amplification mechanisms," IEEE/ASME Transactions on Mechatronics, vol. 15, no. 5, pp. 770-782, 2010.

[17] S. Canfield and M. Frecker, "Topology optimization of compliant mechanical amplifiers for piezoelectric actuators," Structural and Multidisciplinary Optimization, vol. 20, pp. 269-279, 122000.

[18] T. Schlinquer, A. Mohand-Ousaid, and M. Rakotondrabe, "Displacement amplifier mechanism for piezoelectric actuators design using simp topology optimization approach," in IEEE International Conference on Robotics and Automation, May 2018, pp. 4305-4311.

[19] S. Khadraoui, M. Rakotondrabe, and P. Lutz, "Optimal design of piezoelectric cantilevered actuators with guaranteed performances by using interval techniques," IEEE/ASME Transactions on Mechatronics, vol. 19, no. 5, pp. 1660-1668, 2014.

[20] R. Carbonari et al., "Multi-actuated functionally graded piezoelectric micro-tools design: A multiphysics topology optimization approach," Numerical Methods in Engineering, 2008.

[21] B. E. L. G. W. Fabiana, K. Manfredb and S. Fabiand, "Topology optimization of a piezoelectric-mechanical actuator with single- and multiple-frequency excitation," International Journal of Applied Electromagnetics and Mechanics, 2009.

[22] R. J. Wood, E. Steltz, and R. Fearing, "Optimal energy density piezoelectric bending actuators," Sensors and Actuators A: Physical, vol. 119, no. 2, pp. 476-488, 2005.
[23] A. Homayouni-Amlashi, A. M. Ousaid, and M. Rakotondrabe, "Multi directional piezoelectric plate energy harvesters designed by topology optimization algorithm," IEEE Robotics and Automation Letters, 2019.

[24] A. Homayouni-Amlashi, A. Mohand-Ousaid, and M. Rakotondrabe, "Topology optimization of 2dof piezoelectric plate energy harvester under external in-plane force," Journal of Micro-Bio Robotics, pp. 113, 2020.

[25] R. Rakotondrabe, "Performances inclusion for stable interval systemstechniques," ACC, San Francisco CA USA, pp. 4367-4372, 2011.

[26] M. P. Bendsoe and O. Sigmund, Topology Optimization: Theory, Methods and Applications. Springer, Feb. 2004.

[27] M. Kögl and E. C. Silva, "Topology optimization of smart structures: design of piezoelectric plate and shell actuators," Smart materials and Structures, vol. 14, no. 2, p. 387, 2005.

[28] D. Ruiz et al., "Optimal design of a microgripper-type actuator based on AlN/Si heterogeneous bimorph," in Smart Sensors, Actuators, and MEMS VIII, vol. 10246, International Society for Optics and Photonics. SPIE, 2017, pp. 10-19.

[29] K. Chawla, Mechanical Behavior of Materials. Prentice Hall, 1999.

[30] V. Piefort, "Finite element modelling of piezoelectric active structures," Ph.D. dissertation, PhD thesis, Univ. Libre de Bruxelles, 2001.

[31] C.-C. Chung, Microstructural Evolution in Lead Zirconate Titanate (PZT) Piezoelectric Ceramics. Thesis, Univ of Connecticut, 2014.

[32] A. A. N. Standard, "Ieee standard on piezoelectricity," IEEE Transactions on Sonics and Ultrasonics, vol. 31, no. 2, March 1984.

[33] R. Lerch, "Simulation of piezoelectric devices by two- and threedimensional finite elements," IEEE Transactions on Ultrasonics, Ferroelectrics, and Frequency Control, vol. 37, pp. 233-247, May 1990.

[34] E. Andreassen, A. Clausen, M. Schevenels, B. Lazarov, and O. Sigmund, "Efficient topology optimization in matlab using 88 lines of code," Struct Multidiscip Optim, vol. 43, no. 1, pp. 1-16, 2011.

[35] J. Toledo and al., "Design and characterization of in-plane piezoelectric microactuators," Actuators, vol. 6, no. 2, p. 19, 2017.

[36] Z. Luo, W. Gao, and C. Song, "Design of multi-phase piezoelectric actuators," Journal of Intelligent Mateirla Systems and Structures, vol. 21, pp. 1851-1865, 2010.

[37] R. Carbonari, E. Silva, and G. Paulino, "Topology optimization design of functionally graded bimorph-type piezoelectric actuators," Smart Mater. Struct, vol. 16, pp. 2605-2620, 122007.

[38] Z. Kang and X. Wang, "Topology optimization of bending actuators with multilayer piezoelectric material," Smart Materials and Structures, vol. 19, p. 075018, 062010.

[39] L. A. M. Mello et al., "Design of quasi-static piezoelectric plate based transducers by using topology optimization," Smart Materials and Structures, vol. 23, no. 2, p. 025035, jan 2014.

[40] O. Sigmund and J. Petersson, "Numerical instabilities in topology optimization: A survey on procedures dealing with checkerboards, meshdependencies and local minima," Structural optimization, vol. 16, pp. $68-75,1998$

[41] M. P. Bendsøe and O. Sigmund, "Material interpolation schemes in topology optimization," Archive of applied mechanics, vol. 69, no. 910, pp. 635-654, 1999.

[42] P. Bisegna and R. Luciano, "Variational bounds for the overall properties of piezoelectric composites," Journal of the Mechanics and Physics of Solid, vol. 44, no. 4, pp. 583-602, 1996.

[43] J. Y. Li and M. L. Dunn, "Variational bounds for the effective moduli of heterogeneous piezoelectric solids," Philosophical Magazine A, vol. 81, no. 4, pp. 903-926, 2001.

[44] J. E. Ouafi, L. Azrar, and A. A. Aljinaidi, "Analytical and semianalytical modeling of effective moduli bounds: Application to transversely isotropic piezoelectric materials," Journal of Intelligent Material Systems and Structures, vol. 27, no. 12, pp. 1600-1623, 2016.

[45] B. Zheng, C.-J. Chang, and H. C. Gea, "Topology optimization of energy harvesting devices using piezoelectric materials," Structural and Multidisciplinary Optimization, vol. 38, no. 1, pp. 17-23, 2009.

[46] M. Rakotondrabe, "Multivariable classical prandtl-ishlinskii hysteresis modeling and compensation and sensorless control of a nonlinear 2-dof piezoactuator," Nonlinear Dynamics, vol. DOI.10.1007/s11071-0173466-5, 2017.

[47] — Smart materials-based actuators at the micro/nano-scale. Characterization, Control and Applications. Springer Verlag, 2013. 